Editorial

\title{
Subarachnoid Hemorrhage: The Continuum
}

\author{
Harjinder Singh Bhatoe ${ }^{1}$ \\ ${ }^{1}$ Department of Neurosciences, Max Super Speciality Hospital, \\ New Delhi, India \\ Indian J Neurosurg 2015;4:55.
}

Subarachnoid hemorrhage continues to be a major management challenge for neurosurgeons and neurointensivists worldwide. In the Indian scenario, the patients with subarachnoid hemorrhage (SAH) seen in neurosurgery emergency rooms and institutions represent the proverbial tip of the iceberg, the ones who survive the initial ictus and the travel to a neurosurgical center, due to problems in access to medical care and paucity of diagnostic imaging in the rural hinterland. Thus, it is indeed an epidemiological challenge too, to define the extent of the existing problem. At the time of independence in 1947, the problem was considered largely nonexistent in India. Since then, over the past six decades, the magnitude of the problem has dawned, with the realization that many of these patients are not being diagnosed and are missed. The incidence of aneurysmal SAH is reported to be 3 to 4 per 100,000 from the hospital-based data. ${ }^{1}$ There is a paradigm shift, with many centers of excellence offering definitive therapy, microsurgical or endovascular within the first 24 hours of the ictus.

Catastrophe as it is, SAH has multisystemic involvement, and warrants an aggressive emergency neurological and cardiopulmonary resuscitation. Such an attitude, coupled with the early aneurysm repair (by microsurgical or endovascular approach), and a protocolized, advanced multimodality monitoring in a specialized neurointensive unit offers the best milieu for improvement and outcome.

Control of raised intracranial pressure (by cerebrospinal fluid diversion, osmotic diuresis, etc.), optimization of cerebral perfusion and oxygenation, and aggressive medical management to prevent aneurysmal bleeding comprise the initial bulwark in the initial resuscitation, and optimizing the cerebral pathophysiology so that the patient is fit to undergo definitive aneurysmal repair. Postaneurysmal repair, all attention has to be directed to the diagnosis and management of cerebral vasospasm, and prevention of secondary medical complications (dyselectrolytemia, hypotension, hyperglycemia, anemia, and nosocomial sepsis).

The paradigm shift has to occur from a reactive model of detecting secondary insults to identifying targets for goal-directed therapy. The aim is to provide an optimum physiological environment for recovery to the swollen brain, which is under raised pressure, ischemic and deranged micro-, and molecular milieu. The overall mortality in SAH is down to $20 \%$ from $50 \%{ }^{2,3}$ Although the mortality among poor-grade patients (Hunt and Hess grades IV and V) remains high, recent studies have shown that many of these poor-grade patients can recover if treated aggressively by following a protocolized regime. ${ }^{4,5}$ Multimodality monitoring and aggressive, multisystemdirected therapy has followed improved understanding of the pathophysiology of SAH. A concept of "ictal infarction" has channelized the initial management toward aggressive emergency resuscitation, namely, control of intracranial pressure, cardiopulmonary support to optimize cerebral perfusion and oxygenation, blood pressure control, seizure prophylaxis, and proactive measures to prevent rebleeding and secondary brain injury, while establishing a diagnosis by imaging and laying the plans for definitive management.

\section{References}

1 Bhagwati SN. Incidence of subarachnoid hemorrhage from aneurysmal rupture in India. Neurol Med Chir (Tokyo) 1998;38 (Suppl):128-130

2 Komator Ricardo J, Schmidt JM, Starke RM, et al. Resuscititation and critical care of poor-grade subarachnoid hemorrhage. Neurosurgery 2009;64(3):397-410, discussion 410-411

3 Rosengart AJ, Schultheiss KE, Tolentino J, Macdonald RL. Prognostic factors for outcome in patients with aneurysmal subarachnoid hemorrhage. Stroke 2007;38(8):2315-2321

4 Tamargo RJ, Walter KA, Oshiro EM. Aneurysmal subarachnoid hemorrhage: prognostic features and outcomes. New Horiz 1997; 5(4):364-375

5 Mocco J, Ransom ER, Komotar RJ, et al. Preoperative prediction of long-term outcome in poor-grade aneurysmal subarachnoid hemorrhage. Neurosurgery 2006;59(3):529-538, discussion 529-538

\footnotetext{
Address for correspondence Harjinder Singh Bhatoe, MCh, Max Super Speciality Hospital PPG, 108A IP Extension,

New Delhi 110092, India

(e-mail: hsbhatoe@gmail.com).

DOI http://dx.doi.org/ 10.1055/s-0035-1560035. ISSN 2277-954X.
}

(c) 2015 Neurological Surgeons' Society of India
License terms

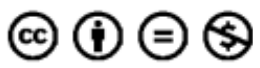

